\title{
STELLAR TIDAL STREAMS IN SPIRAL GALAXIES OF THE LOCAL VOLUME: A PILOT SURVEY WITH MODEST APERTURE TELESCOPES
}

\author{
David Martínez-Delgado ${ }^{1,2}$, R. Jay Gabany ${ }^{3}$, Ken Crawford ${ }^{4}$, Stefano Zibetti ${ }^{1}$, Steven R. Majewski ${ }^{5}$, \\ Hans-Walter Rix ${ }^{1}$, Jürgen Fliri ${ }^{2,6}$, Julio A. Carballo-Bello ${ }^{2}$, Daniella C. Bardalez-Gagliuffi ${ }^{2,7}$, Jorge Peñarrubia ${ }^{8}$, \\ Taylor S. Chonis ${ }^{9}$, Barry Madore ${ }^{10}$, Ignacio Trujillo ${ }^{2}$, Mischa Schirmer ${ }^{11}$, and David A. McDavid 5 \\ ${ }^{1}$ Max Planck Institut für Astronomie, Heidelberg, Germany \\ ${ }^{2}$ Instituto de Astrofísica de Canarias, La Laguna, Spain \\ ${ }^{3}$ Black Bird Observatory, Mayhill, NM, USA \\ ${ }^{4}$ Rancho del Sol Observatory, Modesto, CA, USA \\ ${ }^{5}$ Department of Astronomy, University of Virginia, Charlottesville, VA 22904-4325, USA \\ ${ }^{6}$ GEPI, Observatoire de Paris, Meudon Cedex, France \\ ${ }^{7}$ Massachussetts Institute of Technology, Cambridge, MA, USA \\ ${ }^{8}$ Institute of Astronomy, University of Cambridge, UK \\ ${ }^{9}$ Department of Astronomy, University of Texas, Austin, TX 78712-0259, USA \\ 10 The Observatories of the Carnegie Institution for Science, 813 Santa Barbara Street, Pasadena, CA 91101, USA \\ ${ }^{11}$ Argelander Institut für Astronomie, Universität Bonn, Germany \\ Received 2010 April 11; accepted 2010 July 21; published 2010 September 7
}

\begin{abstract}
Within the hierarchical framework for galaxy formation, minor merging and tidal interactions are expected to shape all large galaxies to the present day. As a consequence, most seemingly normal disk galaxies should be surrounded by spatially extended stellar "tidal features" of low surface brightness. As part of a pilot survey for such interaction signatures, we have carried out ultra deep, wide field imaging of eight isolated spiral galaxies in the Local Volume, with data taken at small $(D=0.1-0.5 \mathrm{~m})$ robotic telescopes that provide exquisite surface brightness sensitivity $\left(\mu_{\lim }(V) \sim 28.5 \mathrm{mag} \operatorname{arcsec}^{-2}\right)$. This initial observational effort has led to the discovery of six previously undetected extensive (to $\sim 30 \mathrm{kpc}$ ) stellar structures in the halos surrounding these galaxies, likely debris from tidally disrupted satellites. In addition, we confirm and clarify several enormous stellar over-densities previously reported in the literature, but never before interpreted as tidal streams. Even this pilot sample of galaxies exhibits strikingly diverse morphological characteristics of these extended stellar features: great circle-like features that resemble the Sagittarius stream surrounding the Milky Way, remote shells and giant clouds of presumed tidal debris far beyond the main stellar body, as well as jet-like features emerging from galactic disks. Together with presumed remains of already disrupted companions, our observations also capture surviving satellites caught in the act of tidal disruption. A qualitative comparison with available simulations set in a $\Lambda$ Cold Dark Matter cosmology (that model the stellar halo as the result of satellite disruption evolution) shows that the extraordinary variety of stellar morphologies detected in this pilot survey matches that seen in those simulations. The common existence of these tidal features around "normal" disk galaxies and the morphological match to the simulations constitutes new evidence that these theoretical models also apply to a large number of other Milky Way-mass disk galaxies in the Local Volume.
\end{abstract}

Key words: dark matter - galaxies: dwarf - galaxies: evolution - galaxies: halos - galaxies: interactions - galaxies: structure

Online-only material: color figure

\section{INTRODUCTION}

Galactic mergers have long been recognized as crucial agents in shaping and evolving galaxies (Toomre \& Toomre 1972). Within the hierarchical galaxy formation framework (e.g., White \& Frenk 1991), dark matter halo mergers are a dominant evolutionary driver on the scale of galaxies. For all mass scales lower than entire galaxy clusters, the merger of two DM halos is followed quickly by the merger of the (stellar) galaxies that had been sitting at the halo's centers (e.g., Kauffmann et al. 1993). The most spectacular manifestations of this process may be major mergers (i.e., the coalescence of galaxies with comparable mass) that usually entails the destruction of any pre-existing stellar disk and may lead to star bursts. Such events have been relatively rare at least since $z \sim 1$, with only a few percent of luminous galaxies being involved in an ongoing major merger at any point in time (e.g., Robaina et al. 2010).
However, minor mergers (i.e., the coalescence of a satellite galaxy and its halo with a much more luminous and massive companion) are expected to be significantly more common (e.g., Cole et al. 2000). Indeed, such minor mergers should remain frequent to the present epoch in a $\Lambda \mathrm{CDM}$ cosmogony. As minor mergers do not destroy pre-existing stellar disks (e.g., Robertson et al. 2006), signs of recent or ongoing minor mergers should be apparent around spirals, the most common type of large galaxy. If the satellite galaxies become tidally disrupted while still in an orbit that extends beyond the stellar body of the larger galaxy companion, then they should form stellar tidal "features," which extend into the halo of the central galaxy.

The observational consequences of this scenario, where the stellar halo of spiral galaxies is essentially comprised of tidal stellar debris from merged satellite galaxies, have been explored by Bullock \& Johnston (2005, BJ05) and others (e.g., Tumlinson 2010; Cooper et al. 2010). Satellites that merged on compact 
Table 1

Observatories and Other facilities

\begin{tabular}{llccccc}
\hline \hline \multicolumn{1}{c}{ Observatory } & \multicolumn{1}{c}{ Location } & Telescope & Focal Ratio & CCD & Scale $\left(\operatorname{arcsec} \operatorname{pixel}^{-1}\right)$ & FOV $(\operatorname{arcmin})^{2}$ \\
\hline BBO & NM, USA & RCO 0.508 m & $f / 8.1$ & STL-11000 & 0.46 & $20.4 \times 30.6$ \\
RdS & CA, USA & RCO 0.508 m & $f / 8.1$ & Alta KAF09000 & 0.58 & $29.3 \times 29.3$ \\
Mrk & South Australia & RC0 0.368 m & $f / 9$ & Alta 16803 & 0.56 & $38.2 \times 38.2$ \\
New Mexico Skies (NMS) & NM, USA & APS 0.160 m & $f / 7$ & STL-11000 & 1.66 & $73.7 \times 110.6$ \\
\end{tabular}

orbits or a long time ago have phase-mixed into a seemingly smooth component by now. In contrast, merger remnants that are only a few dynamical periods old, either because they occurred recently or on orbits with $t_{\text {orbit }} \gtrsim 1 \mathrm{Gyr}$, should leave stellar streams, rings, or plumes as the "fossil record" of their interactions. BJ05 showed that such tidal debris can exhibit a wide range of morphologies and that such distinctive structural features should be common, perhaps ubiquitous around normal disk galaxies. They also showed that most of the features occur at very low surface brightness $\left(\mu_{V} \gtrsim 28.5 \mathrm{mag} \operatorname{arcsec}^{-2}\right)$ and would therefore not be recognizable in traditional images of nearby galaxies.

The Milky Way and the Andromeda galaxy, both resolvable into individual stars so that low surface brightness streams can more readily be seen, show a wealth of (sub-)structure in the stellar distribution of their outskirts. The most spectacular cases are the Sagittarius tidal stream surrounding the Milky Way (e.g., Majewski et al. 2003) and the Great Southern stream around the Andromeda galaxy (e.g., Ibata et al. 2001), which have become archetype fossil records of satellite galaxy mergers. But overall, the stellar halo structure of both galaxies is complex (e.g., Majewski et al. 1996; Belokurov et al. 2006; Bell et al. 2008; McConnachie et al. 2009).

Both simulations and empirical evidence suggest that there is a great deal of galaxy-to-galaxy variation in the level and the epoch of merging and hence variation in the amount and morphology of tidal debris. Therefore, a more than qualitative comparison between the predicted and observed prevalence of stellar debris around disk galaxies requires a much larger sample that necessarily must include galaxies well beyond the Local Group. The current models predict that a survey of between 50 and 100 parent galaxies reaching to a surface brightness of $\sim 30 \mathrm{mag} \mathrm{arcsec}-2$ should reveal many tens of tidal features, perhaps nearly one detectable stream per galaxy (Bullock \& Johnston 2005; Johnston et al. 2008; Cooper et al. 2010).

However, at present the evidence for tidal streams beyond the Local Group is mostly anecdotal, rather than systematic. The first cases of candidate extragalactic tidal stream candidates were reported a decade ago by Malin \& Hadley (1997). Using special contrast enhancement techniques on deep photographic plates, these authors were able to highlight two possible tidal streams surrounding the galaxies M83 and M104. Subsequently, deep CCD images of the nearby, edge-on galaxy NGC 5907 by Shang et al. (1998) revealed an elliptically shaped loop in the halo of this galaxy. This was the most compelling example of an external tidal stream up to now. More recently, very deep images have clearly revealed large-scale, complex structures of arcing loops in the halos of several nearby, NGC galaxies (NGC 5907: Martínez-Delgado et al. 2008; NGC 4013: Martínez-Delgado et al. 2009; NGC 891: Mouhcine et al. 2010) as well as more distant, anonymous galaxies (Forbes et al. 2003).

These results suggest that a more systematic survey for tidal streams in the nearby universe is not only practical but required as a new way to constrain models of galaxy formation.
During the past few years, we have initiated a pilot survey of stellar tidal streams in a selected number of spiral systems using modest telescopes operating at very dark sites. Ultimately, the most basic question we will seek to answer concerns the frequency of stellar streams in the Local Volume. Our aim is to test theoretical predictions by comparing substructure counts from our galaxy sample to cosmological simulations. But the models also make predictions about a number of direct observational characteristics (such as the colors, morphologies, spatial coherence, and extent of halo substructures) that can be tested with the results of our survey.

This paper describes the initial results of our pilot study on eight nearby spiral galaxies. These systems were selected for the study because they were already suspected of being surrounded by diffuse-light over-densities based on data collected from available surveys (e.g., POSS-II; SDSS-I) and previously published deep images posted on the Internet by amateur astronomers. While based on a biased sample of systems preselected for substructures, our pilot study serves as a proof of concept for the intended, more systematic survey of halo substructure around spiral galaxies. It also enabled us to resolve the required observing strategies and data reduction methodologies. The results presented here come from a productive collaboration between amateur and professional astronomers, dedicated to exploiting the scientific potential of modest aperture telescopes.

\section{OBSERVATIONS}

This pilot survey was conducted with three privately owned observatories equipped with modest-sized telescopes located in the USA and Australia (see Table 1). Each observing site features very dark, clear skies with seeing that is routinely below 1".5. These telescopes are manufactured by RC Optical Systems and follow a classic Ritchey-Chretien design. The observatories are commanded with on-site control computers that allow remote operation and control from any global location with highbandwidth Web access. Each observatory uses proven, widely available, remote desktop control software. Robotic orchestration of all observatory and instrument functions, including multiple target acquisition and data runs, is performed using available scripting software. We also make use of a wide field instrument for those galaxies with an especially extended angular size (e.g., NGC 5055; see Figure 1). For this purpose, we have selected the Astro-Physics Starfire (APS) 160EDF6, a short focal length $(f / 7), 16 \mathrm{~cm}$ aperture refractor that provides a field of view (FOV) of $\sim 73.7 \times 110.6$ (see Table 1).

Each telescope is equipped with a commercially available $\mathrm{CCD}$ camera. The primary survey camera of the Black Bird Observatory (BBO) is the SBIG STL-11000, which uses a Kodak KAI-11000M imaging sensor. This sensor consists of a $4008 \times 2672$ pixel array with $9 \times 9 \mu$ m pixels. Some of the other facilities use a 16 megapixel camera manufactured by Apogee Instruments, of Roseville (California). The Rancho del Sol (RdS) observatory uses the Alta-KAFO9000 imaging sensor 

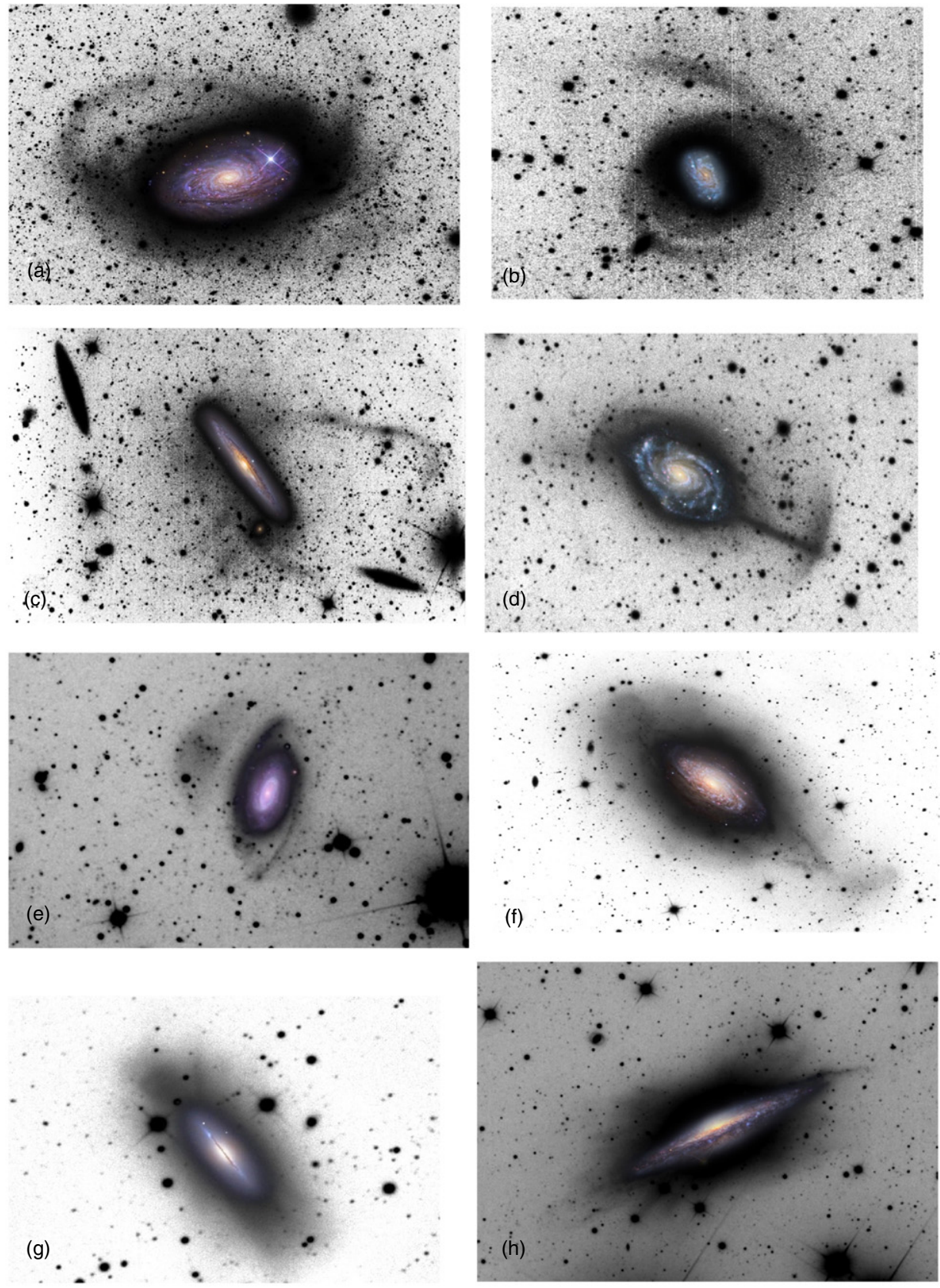

Figure 1. Luminance filter images of nearby galaxies from our pilot survey (see Section 3 for discussion) showing large, diffuse light substructures in their outskirts: (a) a possible Sgr-like stream in Messier 63; (b) giant plumes around NGC 1084; (c) partial tidally disrupted satellites in NGC 4216; (d) an umbrella-shaped tidal debris structure in NGC 4651; (e) an enormous stellar cloud in NGC 7531; (f) diffuse, large-scale and more coherent features around NGC 3521; (g) a prominent spike and giant wedge-shaped structure seen emanating from NGC 5866 (BBO $0.5 \mathrm{~m})$; (h) a strange inner halo in NGC 1055, sprinkled with several spikes of debris (RdS $0.5 \mathrm{~m}$ ). Each panel displays a (linear) super-stretched contrast version of the total image. A color inset of the disk of each galaxy (obtained from data from the same telescope as the luminance images) has been over plotted for reference purposes. In addition, some of the original images were also cropped to better show the most interesting regions around each target.

with $3056 \times 3056$ pixels with $12 \times 12 \mu \mathrm{m}$. The Moorook (MrK) observatory uses a similar CCD camera, the Apogee Alta-KAF16803 with $4096 \times 4096$ pixels and a smaller pixel size $(9 \times 9 \mu \mathrm{m})$.

The tidal stream detection strategy and the procedures used for data acquisition and reduction of the data are the same as those described in our previous papers from this project (Martínez-Delgado et al. 2008, 2009). In summary, this strategy strives for multiple deep exposures of each target using a wide bandpass, high throughput, clear optical filter with a near-IR cutoff, also known as a luminance filter, $L$. The typical cumulative exposure times are in the range of 6-11 hr. Data reduction followed standard techniques described in the afore-mentioned papers. The list of targets, together with the telescopes, and total exposure time used in each case are given in Table 2.

Photometric calibration of the luminance filter $(L)$ images is not currently available. So, to assess their depth and typical quality in terms of background and flat-fielding, we relied on images of six of our galaxies-NGC 1055, NGC 1084, NGC 3521, NGC 4216, NGC 4651, and NGC 5866-obtained by the Sloan Digital Sky Survey (SDSS; York et al. 2000; Data Release 7; Abazajian et al. 2009).

Based on SDSS photometry, we derived photometric equations to convert the $L$-band counts into $g$-band magnitudes. 
Table 2

Observational Data for Galaxies Displayed in Figure 1

\begin{tabular}{clccc}
\hline \hline Target & Facility & Exp. Time $(L)$ & FOV (arcmin) & Features \\
\hline NGC 5055 & NMS 0.16 m & 790 & $37 \times 27$ & $A$ \\
NGC 1084 & BBO 0.51 m & 825 & $15 \times 10$ & $G P$ \\
NGC 4216 & RdS 0.51 m & 1105 & $28 \times 27$ & $P D$ \\
NGC 4651 & BBO 0.51 m & 585 & $14 \times 9$ & $U$ \\
NGC 7531 & Mrk 0.37 m & 600 & $14 \times 10$ & $C l$ \\
NGC 3521 & BBO 0.51 m & 495 & $21 \times 16$ & $M x, U$ ? \\
NGC 5866 & BBO 0.51 m & 315 & $9 \times 7$ & $S p, W$ \\
NGC 1055 & RdS 0.51 m & 400 & $13 \times 9$ & $M x, S p$ \\
\hline
\end{tabular}

Notes. Column 1: NGC number of the target galaxy; Column 2: observatory name and telescope aperture (see Table 1); Column 3: total exposure time in minutes of the luminance image plotted in Figure 1; Column 4: FOV in arcminutes of the zoomed image displayed in Figure 1; Column 5: morphology of the tidal features (see Section 3) recognizable in the outskirts of the galaxy ( $A$, arcs; $G P$, giant plumes; $P D$, partially disrupted satellite; $U$, umbrella; $W$, wedge; $C l$, cloud; $S p$, spike; $M x$, mixed type).

SDSS image mosaics were constructed as described in Zibetti et al. (2009) and high $\mathrm{S} / \mathrm{N}, g-L$ color maps of these galaxies were obtained with ADAPTSMOOTH (Zibetti 2009). Using these maps, we estimated the median zero point and the amplitude of the color terms, which turns out to be of the order of $0.1 \mathrm{mag}$, at most.

There are two main limitations to the depth that can be reached in imaging low-surface brightness features: (1) photon noise and (2) background fluctuations due to flat-field residual, internal reflections, ghosts, scattered light, etc. We estimate the photon noise limit as the surface brightness corresponding to five times rms in $2^{\prime \prime}$ diameter random apertures. For background fluctuations, we estimated the median sky level rms in selected boxes, several tens to hundred arcseconds per side, spread around the galaxies. We find that the typical $2^{\prime \prime}$ diameter detection limit is $27.2 \pm 0.2 \mathrm{mag}_{g} \operatorname{arcsec}^{-2}$, while the typical background fluctuations correspond to $28.5 \pm 0.5 \mathrm{mag}_{g} \operatorname{arcsec}^{-2} .^{12}$ It is worth noting that for the corresponding SDSS $g$-band images we measured 25 and 28.7, respectively. This shows that our images are roughly ten times deeper than the SDSS data in terms of photon statistics and are mainly limited by systematic background uncertainties, which are comparable to those of the SDSS data. This implies that our images have high efficiency in detecting sharp or localized features but background fluctuations hamper our ability to accurately measure smooth diffuse light.

\section{THE RANGE OF TIDAL STREAM MORPHOLOGIES IN THE LOCAL UNIVERSE}

Figure 1 presents recent images of eight nearby spiral galaxies that were found to be surrounded by very low surface brightness structures. In the cases shown, such features were suspected to be present based on various imaging sources including the SDSS-I and POSS-II. Our new observations have enabled the discovery of six morphologically unique tidal stream candidates and reveal the likely tidal nature of numerous faint galaxy structures reported over the last 40 years from inspection of photographic plates. Interestingly, these features do not have $\mathrm{H}$ i counterparts in the available radio surveys (e.g., THINGS: Walter et al. 2008), nor do they display any evidence of recent star formation. This is consistent with features that are predominantly stellar streams

\footnotetext{
$12 g$-Band AB magnitudes are typically 0.3 mag higher than $V$-band Vega magnitudes (see Smith et al. 2002).
}

tidally stripped from dwarf satellite galaxies. The reality of these enormous structures was confirmed through independent images obtained with multiple telescopes given in Table 1; we present the deepest of those images in Figure 1, with the particulars of these images given in Table 2. A detailed investigation of these streams and their properties will be given in a forthcoming contribution (D. Martínez-Delgado et al. 2010, in preparation).

Results of our first forays into low surface brightness imaging included the detection of gigantic looping structures, analogous to the Milky Way's Sagittarius stream, around the spiral galaxy NGC 5907 (Martínez-Delgado et al. 2008). Our image of NGC 5055 here (Messier 63: Figure 1(a)) uncovers a similar, faint, arcing feature in its halo. This presumed tidal stream was first detected in very deep images obtained with the $16 \mathrm{~cm}$ APS apocromatic refractor. This galaxy's giant surrounding structure extends $\sim 30 \mathrm{kpc}$ from the center of NGC 5055 and appears to be unrelated to any faint outer regions of the extended disk that were previously detected by the Galaxy Evolution Explorer space telescope (and also, incidentally, detected in our data). Some insights about this arc were previously reported by van der Kruit (1979) using photographic plates. The position of the stream's progenitor remains unknown-it may be completely disrupted or may lie hidden behind the galaxy's disk.

Our BBO image of the nearby galaxy NGC 1084 (Figure 1(b)) also displays three giant disconnected plumes of similar width extending a large galactocentric distance $(\sim 30 \mathrm{kpc})$ into its halo. Two of these tails emerge in opposite directions from the inner region of the galaxy, while a third one appears completely disconnected from the galaxy. These features were first detected after a close visual inspection of SDSS images. However, it remains difficult to assert if this collection of arcing features is associated with one or several different merger events.

In addition to the remains of presumably long disrupted companions, our data also capture the ongoing tidal disruption of satellite galaxies that are still visible, seen as long tails extending from the progenitor satellite. Perhaps, the most conspicuous examples can be seen in the image of NGC 4216 (Figure 1(c)). This panoramic view of the galaxy shows two satellites with distinct cores and extremely long tails that extend several kiloparsecs into the principal galaxy's halo. The host galaxy also displays a prominent thick disk with several pillars arising from it. The nature of these features (tidal debris or ram pressure signatures) is discussed at length in D. Martínez-Delgado et al. (2010, in preparation).

Among the most conspicuous features found in our survey are coherent structures that resemble an open umbrella and extending tens of kiloparsecs into the host spiral's halo. These spectacular formations are often located on both sides of the principal galaxy and display long narrow shafts that terminate in a giant, partial shell of debris. The most remarkable example so far detected is in NGC 4651, shown in Figure 1(d). This is also the brightest tidal stream detected in our pilot survey (visible even in very short exposure times). The jet-like feature is strikingly coherent and narrow. This feature was previously reported by Vorontsov-Velyaminov (1959) but never interpreted as a stellar tidal stream. Moreover, our deep image shows an additional, spectacular, crescent-shaped shell surrounding the east side of the galaxy that should correspond to the apocenter of the dwarf galaxy progenitor. Interestingly, a possible second arc on the western side of the galaxy can also be seen in this image. This structure is less obvious because it is partially hidden by the galaxy's disk. This suggests that we are observing a moderately inclined structure projected into the halo of NGC 4651. 
The giant, diffuse, cloud-like structure seen in the halo of NGC 7531 (Figure 1(e)) was first reported by Buta (1987) from an inspection of photographic plates. This author classified it as a possible dwarf companion (named A2311.8-4353) in orbit about this spiral galaxy. The report also offered some insights about tidal disruption. Our deeper images clearly reveal the actual shape and extension of this intriguing feature including the presence of small-scale substructuring. Its size is comparable to the disk of the host galaxy. We suspect it might be the shell component of an umbrella-like structure, like that discussed above.

Our images have also reveal the presence of smooth largescale structures that contain coherent substructures spread above and below the plane of several galaxies. These examples (Figures 1(g) and 1(h)) are excellent candidates for systems with "mixed-type" stream ensembles (see Section 4), although alternate origins cannot be rejected. Perhaps the most remarkable system is NGC 1055 (Figure 1(h)). This galaxy displays a clear boxy-shaped inner halo sprinkled with a plethora of coherent "spikes" that seem to emerge from the galaxy's disk. But the most striking examples of spike-like structures that have been detected in our survey thus far are those associated with NGC 5866 (Figure 1(g)). In this system, a collimated structure extends $\sim 8 \mathrm{kpc}$ into the galactic halo. This galaxy also displays more substructure in its "smooth" component, such as a wedge of material in the plane of the disk on its eastern side. The morphology of this disk extension is consistent with the remnants of a recent merger event (e.g., see model F in Figure 6 in Cooper et al. 2010). In a similar way, the diffuse structure detected around NGC 3521 (Figure 1(f)) also contains some discernible substructure, such as an almost spherical cloud of debris visible on its eastern side and a large, more elongated cloud on its western side. Both structures may represent debris shells belonging to an umbrella-like structure, as seen in the image of NGC 4651 (Figure 1(d)), but their fuzzier appearance could suggest that they were accreted much farther in the past.

\section{DISCUSSION AND FUTURE WORK}

Our pilot survey of tidal streams associated with nearby galaxies has revealed that many spiral galaxies in the Local Universe contain significant numbers of gigantic stellar structures that resemble the features expected from hierarchical formation. Although we have only explored a handful of galaxies, our collection already presents a wide spectrum of morphologies for these stellar features. Some of them maybe have analogs in the Milky Way-e.g., (1) great arc-like features that resemble the Milky Way's Sagittarius, Orphan, and Anticenter streams (e.g., Majewski et al. 2003; Belokurov et al. 2006, 2007b; Grillmair 2006) and (2) enormous clouds of debris that resemble our current understandings of the expansive Tri-And and Virgo overdensities and the Hercules-Aquila cloud in the Galactic halo (Rocha-Pinto et al. 2004; Belokurov et al. 2007a; MartínezDelgado et al. 2007; Juric et al. 2008). Our observations also uncover enormous features resembling giant "umbrellas," isolated shells, giant plumes of debris, spike-like patterns emerging from galactic disks, long, tightly coherent streams with a central remnant core and large-scale diffuse forms that are possibly related to the remnants of ancient, fully disrupted satellites.

Remarkably, the diverse morphologies of stellar tidal features detected in our pilot data nearly span the range of morphologies seen in cosmologically motivated simulations. Therefore, they already represent the most comprehensive evidence matching and supporting the detailed hierarchical formation scenario predictions for galaxies similar to the Milky Way. We illustrate this through comparison with the set of 11 available snapshots featuring stellar halo models from BJ05. Each model was constructed with different merger histories in a $\Lambda$ CDM universe and provides an external, panoramic view of surviving tidal debris (see Figure 2) from about 100 satellites. These low-mass systems were "injected" into a central halo potential along orbits whose distributions are consistent with current cosmological models.

Even when limiting the output of the simulations to a surface brightness comparable to our observational limit, the different stream morphologies seen fossilized in these nearby spiral halos (see Figure 1) can easily be identified in snapshots of the model halos as well. This is illustrated by Figure 2, which compares the most conspicuous types of tidal debris detected in our survey with those visible in the model snapshots for three different assembly histories. The abbreviations used in this figure correspond to the following tidal debris types: $A$, great circles; $S p$, Spikes; $U$, umbrellas; $P D$, partial-disrupted satellites; $G P$, giant plumes; $M x$, possibly mixed-type streams. ${ }^{13}$ From an analysis of their models, Johnston et al. (2008, see their Figure 1) concluded that the observed stream morphology is principally dependent upon the progenitor satellite's orbit and accretion epoch. For example, great circle features (like those seen around NGC 5907 and M63; labeled $A$ in Figure 2) apparently arise from near circular orbit accretion events that occurred 6-10 Gyr ago. Straight narrow features with associated shells (e.g., the spikes in NGC 5866 and the umbrella-shaped structure in NGC 4651, labeled " $S p$ " and " $U$," respectively, in Figure 2) were formed in a similar epoch from low-mass satellites in almost radial orbits. Finally, the large-scale diffuse structures observed around NGC 1055 and NGC 5866 (labeled $M x$ in Figure 2) could correspond to the mixed-type category pointed out by these authors, in which case they represent the debris of one accreted satellite that occurred longer than $10 \mathrm{Gyr}$ ago that have had time to fully mix along its orbit.

Figure 2 also illustrates how the stochastic nature of halo formation (Cooper et al. 2010) leads to a large variety of substructures in the outer regions of the galaxies: each halo displays a unique and very complex pattern of stellar debris caused by different defunct companions. This morphological variance among different galaxies should be largest for brighter streams, typically formed by massive and quite recent mergers. This would explain why our modest sample, which was constructed to contain comparatively prominent streams in the Local Universe, reveals such a wide variety of detected streams. Despite the biased sample selection, the results presented here may constitute the first comprehensive observational evidence to support that the predicted great diversity of stellar halos/stream morphologies is actually present in nature.

Encouraged by this pilot survey, we have embarked on the first systematic search for stellar tidal streams in a complete, volume-limited sample of spiral galaxies up to $15 \mathrm{Mpc}$ (i.e., the Local Volume). This will result in the first comprehensive census of stellar stream structures within the Local Volume and it will enable meaningful statistical comparisons with cosmological simulations. The frequency of streams, their stellar populations, and their morphologies will help reveal the nature of the progenitors and lend insights into the underlying structure

\footnotetext{
13 This tight correspondence of observation and theory also holds for the comparison with the more recent simulations by Cooper et al. (2010).
} 
A
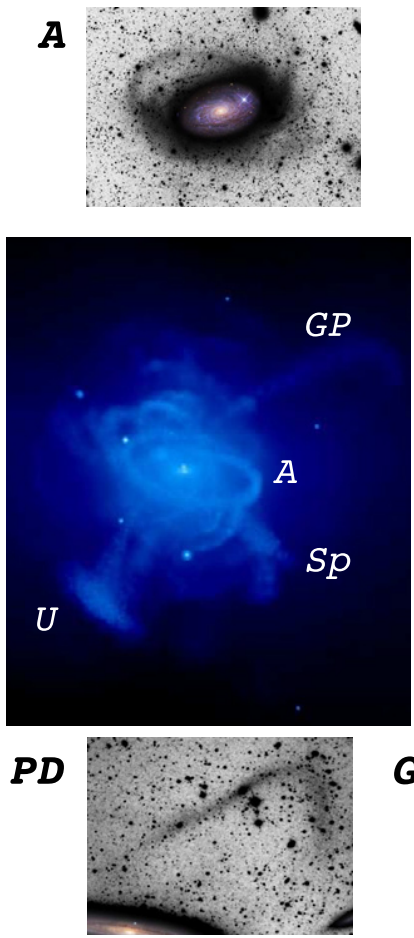

$S p$
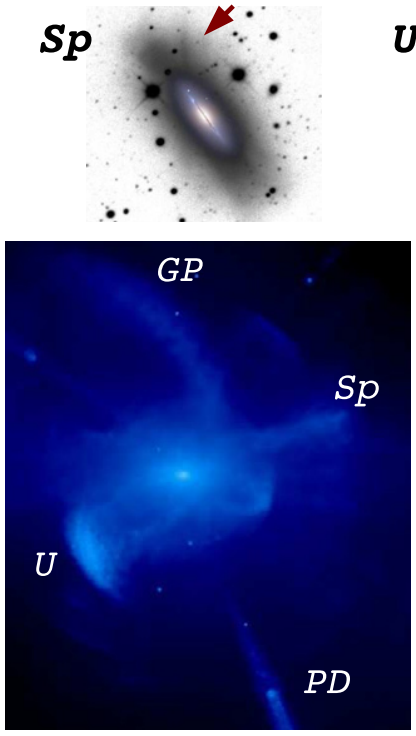

GP

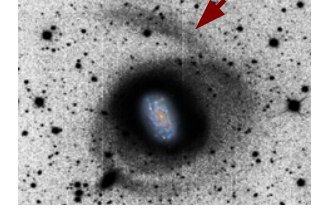

$\boldsymbol{U}$
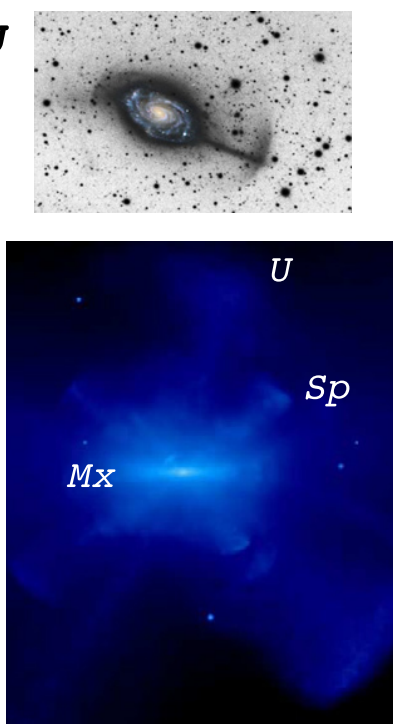

$M x$

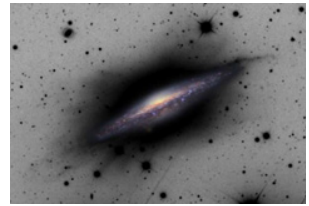

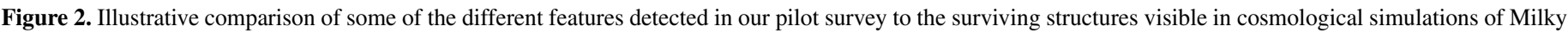

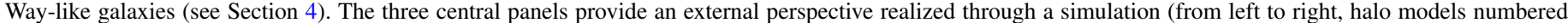

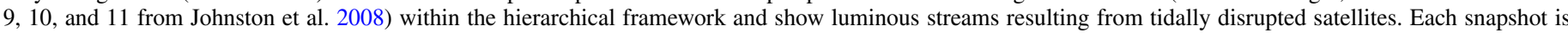

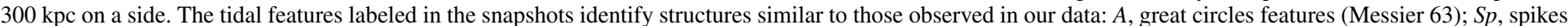

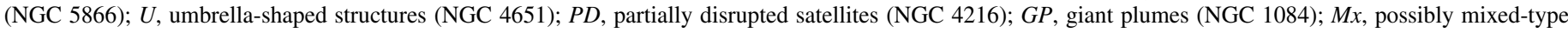
streams (NGC 1055).

(A color version of this figure is available in the online journal.)

and gravitational potential of the massive dark matter halos in which they reside. This will thereby offer a unique opportunity to study the apparently still dramatic last stages of galactic assembly in the Local Universe. In this regard, the survey will be complementary to (and directly inform the interpretation of) local galactic "archaeological" data from resolved galaxies such as M31 and the Milky Way.

We thank K. V. Johnston for providing the models used in this paper and for useful discussion. We also thank $G$. van de Venn, David Valls-Gabaud, Andrew Cooper, and M. A. Gomez-Flechoso for useful discussions. D.M.-D. acknowledges funding from the Spanish Ministry of Education (Ramon y Cajal fellowship and research project AYA 2007-65090) and the Instituto de Astrofisica de Canarias (proyect 3I). S.R.M. appreciates support from NSF grant AST-0807945.

\section{REFERENCES}

Abazajian, K. N., et al. 2009, ApJS, 182, 543

Bell, E., et al. 2008, ApJ, 680, 295

Belokurov, V., et al. 2006, ApJ, 642, L137

Belokurov, V., et al. 2007a, ApJ, 657, 89

Belokurov, V., et al. 2007b, ApJ, 658, 337

Bullock, J. S., \& Johnston, K. V. 2005, ApJ, 635, 931

Buta, R. 1987, ApJS, 64, 1

Cole, S., Lacey, C. G., Baugh, C. M., \& Frenk, C. S. 2000, MNRAS, 319, 168 Cooper, A., et al. 2010, MNRAS, 406, 744

Forbes, D. A., Beasley, M. A., Bekki, K., Brodie, J. P., \& Strader, J. 2003, Science, 301, 1217

Grillmair, C. J. 2006, ApJ, 651, L29
Ibata, R., Irwin, M., Lewis, G., Ferguson, A. M. N., \& Tanvir, N. 2001, Nature, 412,49

Johnston, K. V., Bullock, J. S., Sharma, S., Font, A., Robertson, B. E., \& Leitner, S. N. 2008, ApJ, 689, 936

Juric, M., et al. 2008, ApJ, 673, 864

Kauffmann, G., White, S. D. M., \& Guiderdoni, B. 1993, MNRAS, 264, 201

Malin, D., \& Hadley, B. 1997, PASA, 14, 52

Majewski, S. R., Munn, J. A., \& Hawley, S. L. 1996, ApJ, 459, L73

Majewski, S. R., Skrutskie, M., Weinberg, M., \& Ostheimer, J. 2003, ApJ, 599, 1082

Martínez-Delgado, D., Peñarrubia, J., Gabany, R. J., Trujillo, I., Majewski, S. R., \& Pohlen, M. 2008, ApJ, 689, 184

Martínez-Delgado, D., Peñarrubia, J., Juric, M., Alfaro, E. J., \& Ivezic, Z. 2007, ApJ, 660, 1264

Martínez-Delgado, D., Pohlen, M., Gabany, R. J., Majewski, S. R., \& Palma, C. 2009, ApJ, 692, 955

McConnachie, A. W., et al. 2009, Nature, 461, 66

Mouhcine, M., Ibata, R., \& Rejkuba, M. 2010, ApJ, 714, 12

Robaina, A., Bell, E. F., van der Wel, A., Somerville, R. S., Skelton, R. E., McIntosh, D. H., Meisenheimer, K., \& Wolf, C. 2010, ApJ, 704, 324

Robertson, B., et al. 2006, ApJ, 645, 986

Rocha-Pinto, H. J., Majewski, S. R., Skrutskie, M. F., Crane, J. D., \& Patterson, R. J. 2004, ApJ, 615, 732

Shang, Z., et al. 1998, ApJ, 504, L23

Smith, A. J., et al. 2002, AJ, 123, 2121

Toomre, A., \& Toomre, J. 1972, ApJ, 178, 623

Tumlinson, J. 2010, ApJ, 708, 1398

van der Kruit, P. C. 1979, A\&AS, 38, 15

Vorontsov-Velyaminov, B. A. 1959, Atlas and Catalog of Interacting Galaxies (Moscow: Sternberg Institute, Moscow State Univ.)

Walter, F., Brinks, E., de Blok, W. J. G., Bigiel, F., Kennicutt, R. C., Thornley, M. D., \& Leroy, A. 2008, AJ, 136, 2563

White, S. D. M., \& Frenk, C. S. 1991, ApJ, 379, 52

York, D. G., et al. 2000, AJ, 120, 1579

Zibetti, S. 2009, MNRAS, submitted (arXiv:0911.4956)

Zibetti, S., Charlot, S., \& Rix, H.-W. 2009, MNRAS, 400, 1181 